\title{
Universal intermittent properties of particle trajectories in highly turbulent flows
}

Arnèodo, A.; Benzi, R.; Berg, Jacob; Biferale, L.; Bodenschatz, E.; Busse, A.; Calzavarini, E.; Castaing, B.; Cencini, M.; Chevillard, L.

Total number of authors:

28

Published in:

Physical Review Letters

Link to article, DOI:

10.1103/PhysRevLett.100.254504

Publication date:

2008

Document Version

Publisher's PDF, also known as Version of record

Link back to DTU Orbit

Citation (APA):

Arnèodo, A., Benzi, R., Berg, J., Biferale, L., Bodenschatz, E., Busse, A., Calzavarini, E., Castaing, B., Cencini, M., Chevillard, L., Fisher, R. T., Grauer, R., Homann, H., Lamb, D., Lanotte, A. S., Lévègue, E., Lüthi, B., Mann, J., Mordant, N., ... Yeung, P. K. (2008). Universal intermittent properties of particle trajectories in highly turbulent flows. Physical Review Letters, 100(25), 254504. https://doi.org/10.1103/PhysRevLett.100.254504

\section{General rights}

Copyright and moral rights for the publications made accessible in the public portal are retained by the authors and/or other copyright owners and it is a condition of accessing publications that users recognise and abide by the legal requirements associated with these rights.

- Users may download and print one copy of any publication from the public portal for the purpose of private study or research.

- You may not further distribute the material or use it for any profit-making activity or commercial gain

- You may freely distribute the URL identifying the publication in the public portal 


\title{
Universal Intermittent Properties of Particle Trajectories in Highly Turbulent Flows
}

A. Arnèodo, ${ }^{1}$ R. Benzi,${ }^{2}$ J. Berg, ${ }^{3}$ L. Biferale,,${ }^{4, *}$ E. Bodenschatz,${ }^{5}$ A. Busse,${ }^{6}$ E. Calzavarini, ${ }^{7}$ B. Castaing, ${ }^{1}$ M. Cencini, ${ }^{8, *}$ L. Chevillard, ${ }^{1}$ R. T. Fisher, ${ }^{9}$ R. Grauer,${ }^{10}$ H. Homann, ${ }^{10}$ D. Lamb, ${ }^{9}$ A. S. Lanotte, ${ }^{11, *}$ E. Lévèque, ${ }^{1}$ B. Lüthi, ${ }^{12}$ J. Mann, ${ }^{3}$ N. Mordant,${ }^{13}$ W.-C. Müller,${ }^{6}$ S. Ott,${ }^{3}$ N. T. Ouellette, ${ }^{14}$ J.-F. Pinton, ${ }^{1}$ S. B. Pope,${ }^{15}$ S. G. Roux, ${ }^{1}$ F. Toschi, ${ }^{16,17, *}$ H. Xu, ${ }^{5}$ and P. K. Yeung ${ }^{18}$

(International Collaboration for Turbulence Research)

${ }^{1}$ Laboratoire de Physique de l'École Normale Supérieure de Lyon, 46 allée d'Italie F-69007 Lyon, France

${ }^{2}$ University of Tor Vergata and INFN, via della Ricerca Scientifica 1, 00133 Rome, Italy

${ }^{3}$ Wind Energy Department Ris $\phi$ National Laboratory-DTU, 4000 Roskilde, Denmark

${ }^{4}$ University of Tor Vergata and INFN, Via della Ricerca Scientifica 1, 00133 Rome, Italy

${ }^{5}$ Max Planck Institute for Dynamics and Self-Organization, Am Fassberg 17, D-37077 Goettingen, Germany

${ }^{6}$ Max Planck Institute for Plasma Physics, Garching, Germany

${ }^{7}$ Department of Applied Physics, University of Twente, 7500 AE Enschede, The Netherlands

${ }^{8}$ INFM-CNR, SMC Dipartimento di Fisica, Università di Roma "La Sapienza," Piazzale A. Moro, 2 I-00185 Rome, Italy

${ }^{9}$ Department of Astronomy and Astrophysics, The University of Chicago, Chicago, Illinois 60637, USA

${ }^{10}$ Institute for Theoretical Physics I, Ruhr-University Bochum, 44780 Bochum, Germany

${ }^{11}$ CNR-ISAC, Via Fosso del Cavaliere 100, 00133 Rome and INFN, Sezione di Lecce, 73100 Lecce, Italy

${ }^{12}$ IFU, ETH Zürich, Switzerland

${ }^{13}$ Laboratoire de Physique Statistique de l'Ecole Normal Supérieure CNRS 24 Rue Lhomond, 75231 Paris Cedex 05, France

${ }^{14}$ Haverford College, 370 Lancaster Avenue, Haverford, Pennsylvania 19041, USA

${ }^{15}$ Sibley School of Mechanical and Aerospace Engineering 254 Upson Hall, Cornell University, Ithaca, New York 14853-7501, USA

${ }^{16}$ Istituto per le Applicazioni del Calcolo CNR, Viale del Policlinico 137, 00161 Roma, Italy

${ }^{17}$ INFN, Sezione di Ferrara, Via G. Saragat 1, 44100 Ferrara, Italy

${ }^{18}$ School of Aerospace Engineering, Georgia Institute of Technology, 279 Ferst Drive Atlanta, Georgia 30332-0150, USA

(Received 18 February 2008; published 27 June 2008)

\begin{abstract}
We present a collection of eight data sets from state-of-the-art experiments and numerical simulations on turbulent velocity statistics along particle trajectories obtained in different flows with Reynolds numbers in the range $R_{\lambda} \in$ [120:740]. Lagrangian structure functions from all data sets are found to collapse onto each other on a wide range of time lags, pointing towards the existence of a universal behavior, within present statistical convergence, and calling for a unified theoretical description. ParisiFrisch multifractal theory, suitably extended to the dissipative scales and to the Lagrangian domain, is found to capture the intermittency of velocity statistics over the whole three decades of temporal scales investigated here.
\end{abstract}

DOI: 10.1103/PhysRevLett.100.254504

PACS numbers: 47.27. $-\mathrm{i}$

Understanding the statistical properties of particle tracers advected by turbulent flows is a challenging theoretical and experimental problem [1,2]. It is a key ingredient for the development of stochastic models [3,4], in such diverse contexts as turbulent combustion, industrial mixing, pollutant dispersion, and cloud formation [5]. The main difficulty of Lagrangian investigations, following particle trajectories, stems from the necessity to resolve the wide range of time scales driving different particle behaviors: from the longest $T_{L}$, given by the stirring mechanism, to the shortest $\tau_{\eta}$, typical of viscous dissipation. Indeed the ratio $T_{L} / \tau_{\eta} \sim R_{\lambda}$ grows with the Taylor Reynolds number $R_{\lambda}$ that varies up to few thousands in laboratory flows. Some aspects of Lagrangian statistics have been experimentally measured: particle accelerations [2], velocity fluctuations in the inertial range [6,7], and two-particle dispersion $[8,9]$. Others, connected to the entire range of motions, have long been restricted to numerical simulations [10-14]. A fundamental open question is connected to intermittency, i.e., the observed strong deviations from Gaussian statistics, becoming larger and larger when considering fluctuations at smaller and smaller scales. Additionally, the dependency of velocity statistics at various temporal scales on large scale forcing and boundary conditions is the so-called problem of universality. Thus, universality features are linked to the degree of anisotropy and nonhomogeneities of turbulent statistics [15]. Similar problems have already been explored measuring the velocity fluctuations in the laboratory frame (Eulerian statistics), where clear evidence of universality has been obtained [16]. To build a general theory of turbulent statistics, universality is the first requirement and, if proved, may open the possibility for effective stochastic modeling [17] in many applied situations. This Letter is aimed at inves- 
tigating intermittency and universality properties of velocity temporal fluctuations by quantitatively comparing data obtained from the most advanced laboratory [6-8] and numerical $[10-13,18]$ experiments. The main outcomes of our analysis are twofold. First, we show that data collapse on a common functional form, providing evidence for universality of velocity fluctuations - up to moments currently achievable with high statistical accuracy. At intermediate and inertial scales, data show an intermittent behavior. Second, we propose a stochastic phenomenological modelization in the entire range of scales, using a multifractal description linking Eulerian and Lagrangian statistics.

We analyze the probability distribution of velocity fluctuations at all scales, focusing on moments of these distributions, namely the Lagrangian velocity structure functions (LVSF) of positive integer order $p$ :

$$
S_{i}^{(p)}(\tau)=\left\langle\left[v_{i}(t+\tau)-v_{i}(t)\right]^{p}\right\rangle=\left\langle\left(\delta_{\tau} v_{i}\right)^{p}\right\rangle,
$$

where $i=x, y, z$ are the velocity components along a single particle path, and the average is defined over the ensemble of trajectories. As stationarity and homogeneity are assumed, moments of velocity increments only depend on the time lag $\tau$. In the inertial range, for $\tau_{\eta} \ll \tau \ll T_{L}$, nonlinear energy transfer governs the dynamics. Thus, from a dimensional viewpoint, only the scale $\tau$ and the average energy dissipation rate for unit mass $\epsilon$ should matter for the structure function behavior. The only admissible choice is $S_{i}^{(p)}(\tau) \sim(\epsilon \tau)^{p / 2}$, but it does not take into account the fluctuating nature of energy dissipation. Empirical studies have indeed shown that the tails of the probability density functions of $\delta_{\tau} v$ become increasingly non-Gaussian at decreasing $\tau / T_{L}$. In terms of moments of the velocity fluctuations, intermittency reveals itself in the anomalous scaling exponents, i.e., a breakdown of the dimensional law for which we have that

$$
S_{i}^{(p)}(\tau) \sim \tau^{\xi(p)},
$$

with $\xi(p) \neq p / 2$. Notice that when dissipative effects dominate, typically for scales $\tau \sim \tau_{\eta}$ and smaller, the power-law behavior (2) is no longer valid, and refined arguments have to be employed, as we will see in the following.

The statistics of velocity fluctuations at varying time lag $\tau$ can be quantitatively captured by the logarithmic derivatives of $S_{i}^{(p)}(\tau)$ versus $S_{i}^{(2)}(\tau)$ [19-21]. This defines the local scaling exponents

$$
\zeta_{i}(p, \tau)=\frac{d \log S_{i}^{(p)}(\tau)}{d \log S_{i}^{(2)}(\tau)} .
$$

For statistically isotropic turbulence, all components are equivalent, so that their spread quantifies the degree of anisotropy present in the flow. The $\tau$ dependence of $\zeta_{i}(p, \tau)$ allows for a scale-by-scale characterization of intermittency.

Figure 1 shows the local exponents of order $p=4$ from a collection of eight data sets, see Tables I and II, for different Reynolds numbers. Most of these data sets are new as is the analysis performed here. We focused on the fourth order moment, since it is the highest order achievable with statistical convergence for all data sets. Two observations can be done. First, all data sets show a similar strong variation around the dissipative time $\tau / \tau_{\eta} \sim O(1)$ that depends on the Reynolds number, and then a clear tendency toward a plateau for larger lags $\tau>10 \tau_{\eta}$. Second, all data sets, with comparable Reynolds numbers, agree well in the whole range of time lags. The relative scatter increases only for large $\tau$, due to the combined effects of the lack of statistics, the anisotropy of the flows, and the different values of $R_{\lambda}$. In particular, finite volume effects in experimental particle tracking can produce a small-but systematic - downward shift of the points at long-lag times [21,22]. It is worth noticing that error bars estimated from anisotropic contributions decrease by going to small $\tau$, indicating that isotropy tends to be recovered at sufficiently small scales; i.e., large scale anisotropic contributions become less and less important. In addition, the fact that, at comparable Reynolds numbers, all data sets recover the same behavior by going to smaller and smaller time lags provides a clear indication of Lagrangian universality of the energy cascade. Such an agreement has not been observed before and is comparable with that found for the corresponding Eulerian quantities [16].

The quality of data shown in Fig. 1 opens the possibility of quantitatively testing phenomenological models for LVSF, scale-by-scale. The Parisi-Frisch multifractal (MF) model of the inertial range [23], and its generalization to the dissipative range [24-27], has proved to give a satisfactory description of Eulerian and Lagrangian fluctuations $[14,28-30]$. It is thus appealing to search for a link between Eulerian and Lagrangian statistics [14,28-30], since this points to a unique interpretation of turbulent fluctuations. Moreover, it would reduce the number of free parameters. According to the MF model, Eulerian velocity increments at inertial scales are characterized by a local Hölder exponent $h$, i.e., $\delta_{r} u \sim r^{h}$, whose probability $\mathcal{P}(h) \sim r^{3-D(h)}$ is weighted by the Eulerian fractal dimension $D(h)$ of the set where $h$ is observed [23]. The dimensional relation $\tau \sim r / \delta_{r} u$ bridges Lagrangian fluctuations over a time lag $\tau$ to the Eulerian ones at scale $r$. Following Refs. [27,28], it is shown in Ref. [30] how to extend the MF framework to get a unified description at all time scales for Lagrangian turbulence. Accordingly, Lagrangian increments display a continuous and differentiable behavior at the transition from the dissipative to the inertial range, 


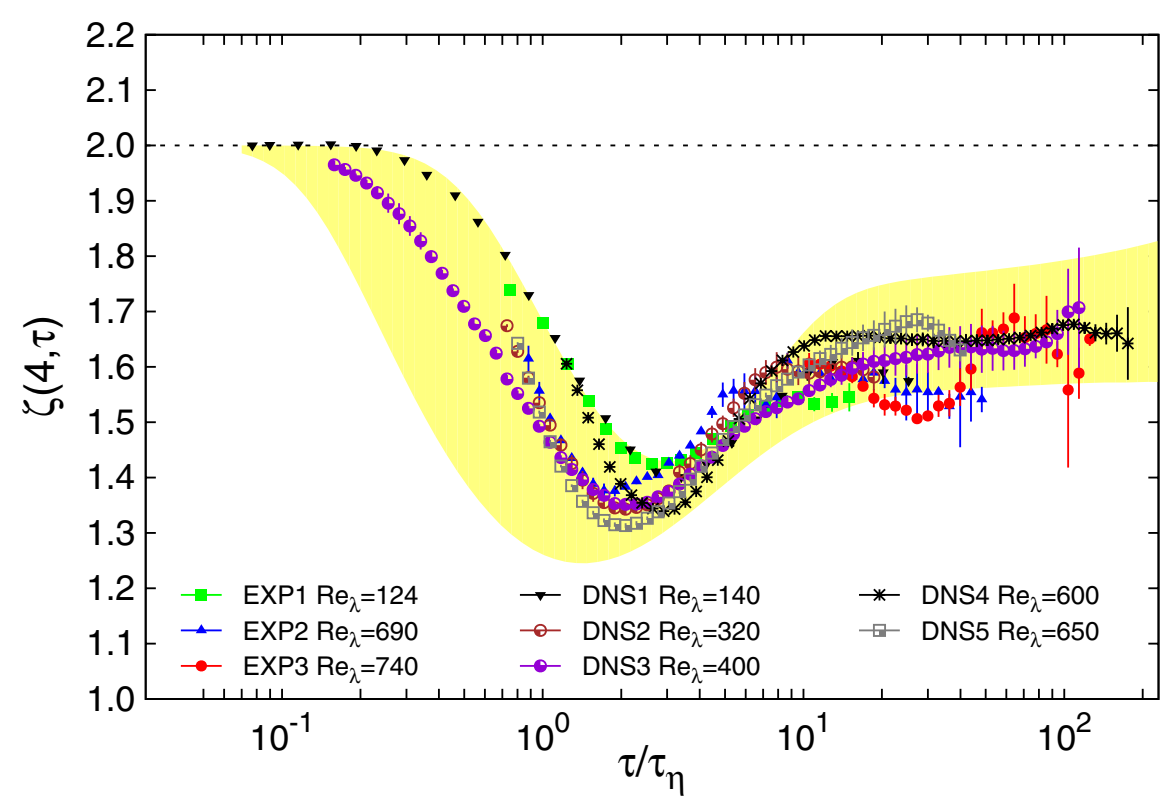

FIG. 1 (color online). Log-lin plot of the fourth order local exponent $\zeta(4, \tau)$ averaged over velocity components, as a function of the normalized time lag $\tau / \tau_{\eta}$. Data sets come from three experiments (EXP) (see Table I) and five direct numerical simulations (DNS) (see Table II). Error bars are estimated from the spread between the three components, except in EXP3 where only two components were measured. Each data set is plotted only in the time range where recognized experimental or numerical limitations are not affecting the results. In particular, for each data set, the largest time lag always satisfies $\tau<T_{L}$. The minimal time lag is set by the highest fully resolved frequency. The shaded area displays the prediction obtained by the MF model by using $D_{\text {lo }}(h)$ or $D_{\text {tr }}(h)$, with $\beta=4$, for a range of $R_{\lambda} \in$ [150:800], comparable with the range of $R_{\lambda}$ in the data. Notice that the MF predictions have been obtained by fixing equal to 7 the multiplicative constant in the definition of $\tau_{\eta}$. The straight dashed line corresponds to the dimensional nonintermittent value $\zeta(4, \tau)=2$, achieved at small time lags where structure functions do become differentiable. Notice that two among the DNS are sufficiently resolved to get the mentioned dimensional scaling in the high frequency limit.

$$
\delta_{\tau} v(h)=V_{0} \frac{\tau}{T_{L}}\left[\left(\frac{\tau}{T_{L}}\right)^{\beta}+\left(\frac{\tau_{\eta}}{T_{L}}\right)^{\beta}\right]^{(2 h-1) / \beta(1-h)},
$$

$\beta$ being a free parameter controlling the crossover around $\tau \sim \tau_{\eta}$, and $V_{0}$ the root mean square velocity. In order to get a prediction for the behavior of the LVSF, given by

$$
\left\langle\left(\delta_{\tau} v\right)^{p}\right\rangle \sim \int d h P_{h}\left(\tau, \tau_{\eta}\right)\left[\delta_{\tau} v(h)\right]^{p},
$$

we have to consider, in (4), the intermittent fluctuations of the dissipative scale $[14,28,30], \tau_{\eta}(h) / T_{L} \sim R_{\lambda}^{2(h-1) /(1+h)}$.

TABLE I. Experiments. By columns: 1: experiment label; 2: Taylor Reynolds number; 3: Kolmogorov time scale $\tau_{\eta}$; 4: measurement volume in unit of the Kolmogorov length scale $\eta$; 5: $N_{\text {tr }}$ total number of Lagrangian trajectories measured; 6: measurement technique-particle tracking velocimetry (PTV) and acoustic Doppler (AD); 7: reference where information on the way the corresponding data set was obtained can be found.

\begin{tabular}{ccrcccc}
\hline \hline EXP & $R_{\lambda}$ & \multicolumn{1}{c}{$\tau_{\eta}(s)$} & Meas. vol. $\left(\eta^{3}\right)$ & $N_{\text {tr }}$ & Tech. & Ref. \\
\hline 1 & 124 & $8.5 \times 10^{-2}$ & $340^{3}$ & $1.6 \times 10^{6}$ & PTV & {$[8]$} \\
2 & 690 & $9 \times 10^{-4}$ & $1700^{3}$ & $6.0 \times 10^{6}$ & PTV & {$[7]$} \\
3 & 740 & $2 \times 10^{-4}$ & $6600^{3}$ & $9.5 \times 10^{3}$ & AD & {$[6]$} \\
\hline \hline
\end{tabular}

The last necessary ingredient is to specify the probability of observing fluctuations of $h$. This is done in analogy to Eq. (4):

$$
P_{h}\left(\tau, \tau_{\eta}\right)=Z^{-1}(\tau)\left[\left(\frac{\tau}{T_{L}}\right)^{\beta}+\left(\frac{\tau_{\eta}}{T_{L}}\right)^{\beta}\right]^{[3-D(h)] / \beta(1-h)},
$$

where $Z$ is a normalizing function [30] and $D(h)$ is the

TABLE II. Direct numerical simulations. By columns: 1: numerical simulation label; 2: Taylor Reynolds number $R_{\lambda} ; 3$ : number of collocation points $N^{3}$; 4: total number of Lagrangian tracers $N_{p}$; 5: characteristic of dissipation-normal viscous terms $(N)$, weakly compressible code $(C)$; 6: interpolation technique for Lagrangian integration - linear interpolation $(L)$, tricubic interpolation $(T)$, cubic splines $(C S)$; 7: reference where information on the way the corresponding data set was obtained can be found.

\begin{tabular}{ccrrccc}
\hline \hline DNS & $R_{\lambda}$ & \multicolumn{1}{c}{$N^{3}$} & \multicolumn{1}{c}{$N_{\text {tr }}$} & Diss. & Tech. & Ref. \\
\hline 1 & 140 & $256^{3}$ & $5 \times 10^{5}$ & $N$ & $T$ & {$[11]$} \\
2 & 320 & $1024^{3}$ & $5 \times 10^{6}$ & $N$ & $T$ & {$[13]$} \\
3 & 400 & $2048^{3}$ & $3 \times 10^{5}$ & $N$ & $L$ & {$[10]$} \\
4 & 600 & $1856^{3}$ & $1.6 \times 10^{7}$ & $C$ & $L$ & {$[18]$} \\
5 & 650 & $2048^{3}$ & $4 \times 10^{5}$ & $N$ & CS & {$[12]$} \\
\hline \hline
\end{tabular}


fractal dimension of the support of the exponents $h$. Once the Reynolds number has been specified, we are left with two parameters, the exponent $\beta$ and a multiplicative constant in the definition of $\tau_{\eta}$, while the function $D(h)$ comes from the knowledge of the Eulerian statistics. Eulerian velocity structure functions (EVSF) have been measured in the past two decades (see Ref. [16] for a data collection) providing a way to estimate the function $D(h)$ based on empirical data. Many functional forms have been proposed in the literature that are consistent with data, up to statistical uncertainties. Eulerian velocity statistics can be measured in terms of longitudinal or transverse fluctuations. Fluid velocity along particle paths is naturally sensitive to both kinds of fluctuations. We thus evaluated the LVSF in (5) using the fractal dimensions $D_{\mathrm{lo}}(h)$ and $D_{\text {tr }}(h)$ obtained by longitudinal [16] and transverse [31] moments of Eulerian fluctuations, respectively.

The shaded area in Fig. 1 represents the range of variation of the MF prediction computed from $D_{\mathrm{lo}}(h)$ or $D_{\mathrm{tr}}(h)$, measured in the Eulerian statistics (see below), and at changing Reynolds numbers. This must be interpreted as our uncertainty. The prediction works very well: all data fall within the shaded area. The role of the parameters is clear. Changing $\beta$ modifies the sharpness and shape of the dip region at $\tau_{\eta}$ - the larger $\beta$ the more pronounced the dip; while changing the multiplicative constant in the definition of $\tau_{\eta}$ has no effect on the curve shape, but it rigidly shifts the whole curve along the time axis. Increasing the Reynolds number $R_{\lambda}$, the flat region at large lags develops a longer plateau. In the limit $R_{\lambda} \rightarrow \infty$ the MF model predicts $\zeta(4) \simeq 1.71$ from $D_{\mathrm{lo}}(h)$ and $\zeta(4) \simeq 1.59$ from $D_{\text {tr }}(h)$ statistics.

For the Eulerian $D(h)$, we used the following logPoisson [23] functional form,

$$
D(h)=\frac{3\left(h-h^{*}\right)}{\log (\gamma)}\left[\log \left(\frac{3\left(h^{*}-h\right)}{d^{*} \log (\gamma)}\right)-1\right]+3-d^{*}
$$

Different couples of parameters $\left(h^{*}, \gamma\right)$ have been chosen to fit longitudinal and transverse Eulerian fluctuations. The parameter $d^{*}=\left(1-3 h^{*}\right) /(1-\gamma)$ is fixed by imposing the exact relation for third order EVSF. For the longitudinal exponents [16], we used $\left(h_{\mathrm{lo}}^{*}=1 / 9, \gamma_{\mathrm{lo}}=2 / 3\right)$ [23]. For the transverse exponents, we used $\left(h_{\mathrm{tr}}^{*}=1 / 9, \gamma_{\mathrm{tr}}=1 / 2\right)$, which fits the data in Ref. [31] (see [32] for details.)

This comprehensive comparison of the best available experiments and direct numerical simulations provides strong evidence of the universality of Lagrangian statistics. One important open question is the effect of a mean flow, as in turbulent jets [33] and wall bounded turbulence, where strong persistence of anisotropy may break the recovery of small-scale universality. We showed that a multifractal description is in good agreement with data, even in the dissipative range where intermittency is significantly increased. The multifractal description captures the intermittency at all scales with only a few parameters, independent of the Reynolds number. This is the universal feature of Lagrangian turbulence revealed by this study. There exists a long debate on the statistical importance of vortex filaments around dissipative time and length scales $[23,34]$. Simulations $[10,20,35]$ show that the dip region for $\tau \sim \tau_{\eta}$ can be depleted (enhanced) by decreasing (increasing) the probability of particles being trapped in vortex filaments. The multifractal model is able to capture the intermittency around $\tau_{\eta}$ with the help of the free parameter $\beta$. Different values of $\beta$ should then correspond to different statistical weights of vortex filaments along particle trajectories. Only further advances in both experimental techniques and numerical power will allow us to test the same questions here addressed also for the higher order statistics.

Helpful discussions with U. Frisch and L. P. Kadanoff are gratefully acknowledged. We thank the DEISA Consortium (co-funded by the EU, FP6 project 508830) for support within the DEISA Extreme Computing Initiative. L. B., M. C., A. S. L., and F. T. thank CINECA (Bologna, Italy) for technical support.

*ictr.rome@roma2.infn.it

[1] G. Falkovich and K. R. Sreenivasan, Phys. Today 59, 43 (2006).

[2] A. La Porta et al., Nature (London) 409, 1017 (2001).

[3] S. B. Pope, Turbulent Flows (Cambridge University Press, Cambridge, England, 2000).

[4] A. G. Lamorgese, S. B. Pope, P. K. Yeung, and B. L. Sawford, J. Fluid Mech. 582, 423 (2007).

[5] R. A. Shaw, Annu. Rev. Fluid Mech. 35, 183 (2003).

[6] N. Mordant, P. Metz, O. Michel, and J.-F. Pinton, Phys. Rev. Lett. 87, 214501 (2001).

[7] H. Xu et al., Phys. Rev. Lett. 96, 024503 (2006).

[8] J. Berg et al., Phys. Rev. E 74, 016304 (2006).

[9] M. Bourgoin et al., Science 311, 835 (2006).

[10] L. Biferale et al., Phys. Fluids 17, 021701 (2005).

[11] N. Mordant, E. Lévèque, and J.-F. Pinton, New J. Phys. 6, 116 (2004).

[12] P. K. Yeung, S. B. Pope, and B. L. Sawford, J. Turbul. 7, 1 (2006).

[13] H. Homann et al., J. Plasma Phys. 73, 821 (2007).

[14] L. Biferale et al., Phys. Rev. Lett. 93, 064502 (2004).

[15] L. Biferale and I. Procaccia, Phys. Rep. 414, 43 (2005).

[16] A. Arnèodo et al., Europhys. Lett. 34, 411 (1996).

[17] B. L. Sawford, Phys. Fluids A 3, 1577 (1991).

[18] R. T. Fisher et al., IBM J. Res. Dev. 52, 127 (2008).

[19] R. Benzi et al., Phys. Rev. E 48, R29 (1993).

[20] I. Mazzitelli and D. Lohse, New J. Phys. 6, 203 (2004).

[21] L. Biferale et al., Phys. Fluids 20, 065103 (2008).

[22] S. Ott and J. Mann, New J. Phys. 7, 142 (2005).

[23] U. Frisch, Turbulence: The Legacy of A. N. Kolmogorov (Cambridge University Press, Cambridge, England, 1995).

[24] G. Paladin and A. Vulpiani, Phys. Rev. A 35, 1971 (1987).

[25] M. Nelkin, Phys. Rev. A 42, 7226 (1990). 
[26] U. Frisch and M. Vergassola, Europhys. Lett. 14, 439 (1991).

[27] C. Meneveau, Phys. Rev. E 54, 3657 (1996).

[28] M. S. Borgas, Phil. Trans. R. Soc. A 342, 379 (1993).

[29] G. Boffetta, F. De Lillo, and S. Musacchio, Phys. Rev. E 66, 066307 (2002).

[30] L. Chevillard et al., Phys. Rev. Lett. 91, 214502 (2003).

[31] T. Gotoh, D. Fukayama, and T. Nakano, Phys. Fluids 14, 1065 (2002).

[32] The functions $D_{\mathrm{lo}}(h)$ and $D_{\mathrm{tr}}(h)$ are convex; the strongest fluctuations, corresponding to the smallest Hölder exponent $h$, are realized for $h=h_{\mathrm{lo}}^{*}$ and $h_{\mathrm{tr}}^{*}$, respectively. The maxima of fractal dimensions, $D_{\mathrm{lo}}\left(h_{\mathrm{lo}}^{\max }\right)=D_{\mathrm{tr}}\left(h_{\mathrm{tr}}^{\max }\right)=$ 3 , are attained for $h_{\mathrm{lo}}^{\max }=0.382$ and $h_{\mathrm{tr}}^{\max }=0.57$. Since only the range $h^{*} \leq h \leq h^{\text {max }}$ is relevant to the fit of EVSF of positive order [23], the extremes of integration in Eq. (5) have been set in the interval $\left[h_{10}^{*}, h_{\mathrm{lo}}^{\max }\right]$ and $\left[h_{\mathrm{tr}}^{*}, h_{\mathrm{tr}}^{\max }\right]$, respectively. Modifying $h^{\max }$ alters the MF prediction for $\tau \sim T_{L}$, but does not change the behavior of the curves in the inertial range.

[33] P. Gervais et al., Exp. Fluids 42, 371 (2007).

[34] S. Douady, Y. Couder, and M. E. Brachet, Phys. Rev. Lett. 67, 983 (1991).

[35] J. Bec et al., Phys. Fluids 18, 081702 (2006). 